\title{
Der neue Pflegebedürftigkeitsbegriff: Die Empfehlungen des Experten- beirats zur konkreten Ausgestaltung seiner Einführung revisited
}

\section{HEIKE HOFFER}

Heike Hoffer ist Wissenschaftliche Referentin im Deutschen Verein für öffentliche und private Fürsorge e.V., Berlin und abgeordnet an das Bundesministerium für Gesundheit in Berlin. Sie leitete dort die Geschäftsstelle des Expertenbeirats zur konkreten Ausgestaltung des neuen Pflegebedürftigkeitsbegriffs
Die Rezeption des im Juni 2013 an den Bundesminister für Gesundheit übergebenen „Bericht des Expertenbeirats zur konkreten Ausgestaltung des neuen Pflegebedürftigkeitsbegriffs" war gemischt. Das Vorliegen eines stimmigen Konzepts wurde begrüßt; das vermeintliche Fehlen einer „magischen Zahl“, d.h. wie viele Milliarden Euro die Umsetzung denn nun kosten solle, beklagt. Der Beitrag stellt zentrale Ergebnisse des Expertenberichts im Lichte der Diskussionen des Beirats dar. Er verdeutlicht, warum die leistungsrechtliche Gleichbehandlung körperlich, kognitiv und psychisch Pflegebedürftiger die Aufgabe des Faktors „Zeit" als Bemessungsgrundlage erfordert. Zugleich macht er deutlich, warum der Glaube an eine „magische Zahl“ nicht weiterhilft und der Verzicht auf eine Nennung aus fachlicher Sicht überzeugend ist. Es wird klargestellt, dass die Pflege der Zukunft neben der hierfür erforderlichen Einführung des neuen Pflegebedürftigkeitsbegriffs auch die Lösung anderer offener Fragen erfordert.

\section{Einleitung}

Am 27. Juni 2013 hat der „Expertenbeirat zur konkreten Ausgestaltung des neuen Pflegebedürftigkeitsbegriffs“" dem Bundesminister für Gesundheit, Daniel Bahr, seinen Abschlussbericht ${ }^{1}$ übergeben. Kern der Arbeiten des Expertenbeirats war die Frage, wie der in den Jahren 2006-2009 entwickel-
1 Der Bericht des Expertenbeirats zur konkreten Ausgestaltung des neuen Pflegebedürftigkeitsbegriffs, nachfolgend zitiert als "Bericht des Expertenbeirats, S. ...", ist samt Anlagen (Studienergebnisse und Expertisen) auf der Webseite des Bundesministeriums für Gesundheit unter www.bundesgesundheitsministerium.de/Beiratsbericht-Pflegebeduerftigkeitsbegriff verfügbar. 
$t^{2}$ neue Pflegebedürftigkeitsbegriff, der körperliche, kognitive und psychische Störungen erstmals gleichermaßen berücksichtigt, konkret gesetzlich eingeführt werden kann. Die Empfehlungen des Expertenbeirats wurden in 15-monatiger Arbeit von den verschiedenen Gremien ${ }^{3}$ des Expertenbeirats mit insgesamt 83 Expertinnen und Experten aus allen Bereichen der Pflege in insgesamt 67 Sitzungen entwickelt. Der Abschlussbericht enthält über $50 \mathrm{Emp}$ fehlungen und Hinweise zu den Veränderungs- und Anpassungsbedarfen, die sich nach Einschätzung des Expertenbeirats in der Gesetzlichen Pflegeversicherung im Zuge der seit vielen Jahren geforderten gesetzlichen Neudefinition des Begriffs der Pflegebedürftigkeit ergeben. Zugleich stellt er eine stimmige Grundlage für die geplante und zur Erreichung seiner Ziele erforderliche Einführung des Begriffs dar.

Rund um den Übergabetermin wurde der Beiratsprozess intensiv durch die Medien begleitet. Die öffentliche Diskussion wurde vorrangig durch zwei zentrale Fragen bestimmt: Welche Mehrausgaben sich für die soziale Pflegeversicherung aus der geplanten Einführung ergeben (können) und warum der Expertenbeirat mit der Beantwortung der offenen Fragen betraut wurde.

Mit der ersten Frage wird die fachlich umfassende und komplexe Arbeit des Expertenbeirats auf eine politisch, nicht fachlich zu setzende "magische Zahl“ verkürzt. Dies zeigt, dass viele wesentliche Themen des Expertenbeirats bei der Rezeption überlesen wurden. Daher ist es ein Ziel dieses Beitrags, die aus Sicht der Autorin ${ }^{4}$ wesentlichen Aussagen des Beiratsberichts - teilweise im Spiegel des jeweiligen Diskussionsverlaufs - in ihrer Relevanz für die Pflegebedürftigen zusammenfassend zu erläutern.

Zudem wird dieser Beitrag - in dem Versuch, eingangs auch die zweite öffentlich formulierte Frage zu beantworten - auf die Aufgabe und Rolle des Expertenbeirats in dem Prozess zur Neudefinition des Pflegebedürftigkeitsbegriffs eingehen. Weiterhin wird der zeitliche Horizont dargestellt, der nach Einschätzung des Expertenbeirats für die Einführung veranschlagt werden muss. Der Beitrag schließt mit einer kurzen Zusammenfassung und einem Fazit zum Bericht des Expertenbeirats.

\section{Konzeption und Erforderlichkeit des neuen Pflegebedürftigkeitsbegriffs}

Pflegebedürftigkeit ist ein komplexes Konstrukt, das sich pflegewissenschaftlich erst aus der Gesamtschau vieler Einzelaspekte erschließt. Zwar gibt es international eine weitgehende Übereinkunft darüber, welche Grundaspekte nach einem modernen pflegewissenschaftlichen Verständnis Pflegebedürftigkeit begründen. Nach diesem Verständnis ist pflegebedürftig, wer infolge fehlender personaler Ressourcen, mit denen körperliche oder psychische Schädigungen, die Beeinträchtigung körperlicher, kognitiver oder psychischer Funktionen oder gesundheitlich bedingte Belastungen und Anforderungen kompensiert oder bewältigt werden können, dauerhaft oder vorübergehend zu selbständigen Aktivitäten im Lebensalltag, selbständiger Krankheitsbewältigung oder selbständiger Gestaltung von Lebensbereichen und sozialer Teilhabe nicht in der Lage und daher auf personelle Hilfe angewiesen ist. ${ }^{5}$ Durch diese Definition wird bereits deutlich, dass die für die Feststellung von Pflegebedürftigkeit zu berücksichtigenden Einzelaspekte vielfältig sein können.

Bei dem neuen Begriff der Pflegebedürftigkeit, der vom früheren Beirat im Jahre 2009 zur Umsetzung in der Gesetzlichen Pflegeversicherung empfohlen wurde, werden diese Einzelaspekte durch das in diesem Zuge entwickelte, neue Begutachtungsassessment (NBA) konkretisiert und operationalisiert. Mit dem NBA werden Pflegebedürftige zukünftig statt in drei Pflegestufen in fünf Pflegegrade eingestuft. Das NBA ist modular aufgebaut; die Zuordnung zu einem Pflegegrad ergibt sich aus der Gesamtsumme der in den Modulen erreichten Punkte. Die Festlegung, welche Aspekte der Pflegebedürftigkeit im NBA enthalten sein sollen und wie die Module des NBA zu gewichten sind, wurde nicht allein aus pflegewissenschaftlicher Sicht entwickelt, sondern berücksichtigt bereits die spezifische Rolle der Gesetzlichen Pflegeversicherung im System der deutschen Sozialversicherungen und das Ziel, Pflegebedürftigkeit als Zugangsvoraussetzung für eine sozial(versicherungs)rechtliche
Leistung zu definieren. Damit wird deutlich, dass - wie auch bei der Gestaltung der Pflegeversicherung und des Sozialleistungssystems insgesamt neben fachlichen auch normative, d.h. Wertentscheidungen die Entwicklung des NBA zentral mit geprägt haben. Diese Wertentscheidungen haben die Arbeit des Expertenbeitrags wesentlich mit bestimmt.

Die zentrale Wertentscheidung für die Entwicklung des neuen Pflegebedürftigkeitsbegriffs war, Pflegebedürftige mit körperlichen, kognitiven und psychischen Beeinträchtigungen zukünftig

\section{eben fachlichen haben auch Wertentscheidungen, die Entwicklung des NBA zentral eprägt.}

in der Gesetzlichen Pflegeversicherung gleich zu behandeln. Damit sollen die Unterschiede im Leistungsbezug von pflegebedürftigen Menschen mit vorrangig körperlichen Beeinträchtigungen und pflegebedürftigen Menschen, die vorrangig kognitive (z.B. demenzielle) oder psychische Beeinträchtigungen haben, beendet werden. Es wäre ein historischer Schritt: Dass die Definition der Pflegebedürftigkeit kognitive und psychische Beeinträchtigungen und ihre Folgen (z.B. ein deutlich erhöhter allgemeiner Beaufsichtigungs- und Betreuungsbedarf) nur unzureichend erfasst und damit Menschen mit diesen Beeinträchtigungen benachteiligt, war schon lange vor Etablierung der Gesetzlichen Pflegeversicherung im Recht der Sozi-

2 Vgl. hierzu den Bericht des Beirats zur Überprüfung des Pflegebedürftigkeitsbegriffs (2009) sowie den Umsetzungsbericht des Beirats (2009). Für Berichte und Expertisen zum Beirat 2009 wird auf das Literaturverzeichnis des Berichts des Expertenbeirats, S. 113 f., verwiesen.

3 Für eine Übersicht siehe Bericht des Expertenbeirats zur konkreten Ausgestaltung des neuen Pflegebedürftigkeitsbegriffs (2013), S. 18, dort Abb. 1: Gremienstruktur des Expertenbeirats

4 Die Autrorin hat diesen Text noch in ihrer Funktion als Leiterin der Geschäftsstelle des Expertenbeirats verfasst. Die Aussagen in diesem Text geben die persönliche Meinung der Autorin wieder. Sie stellen keine Positionen des Deutschen Vereins für öffentliche und private Fürsorge e.V. oder des Bundesministeriums für Gesundheit dar.

5 Vgl. Bericht des Expertenbeirats, S. 11. 
alhilfe bekannt und wird ebenso lange kritisiert. $^{6}$

Eine wesentliche Konsequenz dieser Wertentscheidung ist, dass Pflegebedürftigkeit - anders als gegenwärtig in den $\int \mathbb{S} 14,15$ SGB XI vorgesehen - nicht mehr über den Faktor „Zeit“ bemessen werden kann und soll, denn der zeitliche Pflege- und Betreuungsbedarf, der aus körperlichen, kognitiven und psychischen Beeinträchtigungen resultiert, ist sehr heterogen und reflektiert den Schweregrad der Beeinträchtigung nur unzureichend und auch nur in Teilbereichen. Mit anderen Worten: Mit einer Zeitmessung ist die Gleichbehandlung gleichschwer betroffener Pflegebedürftiger, deren Pflegebedarf aus unterschiedlichen Gründen (körperlich, kognitiv oder psychische Beeinträchtigung) besteht und der zudem - insbesondere in der häuslichen Versorgung - von einer Vielzahl an Faktoren, die mit der Schwere der Pflegebedürftigkeit nichts zu tun haben, abhängt, nicht erreichbar.

Nach Einschätzung des Expertenbeirats ist die Notwendigkeit, den neuen Pflegebedürftigkeitsbegriff einzuführen, auch durch die im Laufe der Jahre mit verschiedenen Änderungsgesetzen, zuletzt durch das Pflege-NeuausrichtungsGesetz $(\mathrm{PNG})^{7}$, in die Gesetzlichen Pflegeversicherung eingeführten, zusätzlichen Leistungen für Personen mit „eingeschränkter Alltagskompetenz" (vgl. \45a SGB XI) nicht entfallen, da diese die bestehenden Ungerechtigkeiten nicht beseitigt, sondern nur verringert haben. ${ }^{8}$ Konsequenter Weise gelten die mit dem PNG zusätzlich eingeführten Leistungen des $\ 123$ SGB XI und die Möglichkeit, Betreuung als Sachleistung in Anspruch zu nehmen $(\mathbb{S} 124$ SGB XI) daher auch nur bis zum Inkrafttreten eines Gesetzes zur Einführung eines neuen Pflegebedürftigkeitsbegriffs.

Neben dieser zentralen Zielsetzung bieten der neue Pflegebedürftigkeitsbegriff und das NBA weitere Vorteile (z.B. die verbesserte Begutachtung der Beeinträchtigungen von Kindern oder die verbesserte Berücksichtigung präventions- und rehabilitationsrelevanter Aspekte) ${ }^{9}$, die Ausgangspunkt für Verbesserungen und die Weiterentwicklung der Pflegeplanung und -organisation der Gesetzlichen Pflegeversicherung sein können. Der neue Pflegebedürftigkeitsbegriff ist daher weit mehr als die - auch für sich genommen sehr be- deutsame - Gleichstellung verschiedener Personengruppen in der Gesetzlichen Pflegeversicherung: Er ist eine wesentliche Grundlage für eine pflegewissenschaftlich fundierte Weiterentwicklung

\section{Der neue Pflegebedürftigkeits- begriff ist eine wesentliche Grundlage für eine pflegewissenschaftlich fundierte Weiterentwicklung der Pflege- versicherung.}

Die Einführung des neuen Pflegebedürftigkeitsbegriffs und die damit verbundenen Zielsetzungen erforderten eine Betrachtung von Grundsatzfragen und zugleich eine umfassende Analyse von Einzelaspekten der Pflegeversicherung, und zwar in fachlicher, rechtlicher und organisatorischer Hinsicht mit Blick auf die Auswirkungen auf die Pflegebedürftigen, Pflegekräfte, Leistungserbringer und Leistungsträger. Um dieser komplexen und zugleich umfassenden Aufgabe gerecht zu werden, hat der Expertenbeirat die Fragestellungen in vier

der Gesetzlichen Pflegeversicherung hin zu einer teilhabe-, präventions- und nutzerorientierten Pflege.

\section{Aufgabe und Rolle des Expertenbeirats zur konkreten Ausgestaltung des neuen Pflegebedürftigkeitsbegriffs}

Der Expertenbeirat hatte die Aufgabe, konkrete Fragen des Ministers ${ }^{10}$ zur Einführung des neuen Pflegebedürftigkeitsbegriffs zu bearbeiten. Für die Einordnung dieser Aufgabe sind folgende Besonderheiten anzumerken, die gegenüber der Arbeit anderer Beiräte in der Politik bestanden: Einerseits knüpfte der Expertenbeirat an seine eigene Tradition an, aber mit veränderter Arbeitsweise. So hatte es in überwiegend gleicher (was die Einbeziehung von Fachorganisationen angeht) Besetzung bereits in den Jahren 2006-2009 einen Beirat zur Überprüfung des Pflegebedürftigkeitsbegriffs gegeben, der die pflegewissenschaftliche Entwicklung des neuen Verständnisses von Pflegebedürftigkeit und des neuen Begutachtungsassessments (NBA) fachlich begleitet hatte. Dieser Beirat hat im Rahmen der Entwicklung und Grundlegung des neuen Begriffs und des NBA bereits grundlegende Empfehlungen ausgesprochen. Die Erarbeitung der fachlichen Grundlagen lag jedoch - auf der Basis eines entsprechenden Auftrags des Bundesministeriums für Gesundheit - vorrangig bei den beteiligten Wissenschaftler/innen. Dem gegenüber wurden im Expertenbeirat die wesentlichen fachlichen Arbeiten durch den Beirat selbst erledigt bzw. durch Beauftragung von Wissenschaftler/innen konzeptionell gesteuert.
Arbeitsgruppen und weiteren zuarbeitenden Teams und Einzelsachverständigen, teilweise ergänzt durch Studien und Expertisen, bearbeitet. Angesichts der Beteiligung von 37 Fachorganisationen bzw. -institutionen und der notwendig komplexen Arbeitsstruktur, koordiniert durch die Koordinierungsgruppe und fachlich unterstützt sowie zusammengeführt durch die Geschäftsstelle, ist die Erarbeitung und Verabschiedung des Berichts in nur 15 Monaten eine nicht zu unterschätzende Leistung.

Andererseits ist der Einsatz des Expertenbeirats in dieser Funktion ein gewisses Novum. Beiräte, die der Politik zu einer konkreten Frage zuarbeiten, sind nicht selten. Dabei bestehen Beiräte jedoch häufig zentral aus Wissenschaftler/ innen, die auf diesem Wege ihre Expertise in einen politischen Prozess einbringen, um hierfür die fachlichen Grundlagen aus wissenschaftlicher Sicht zu liefern. Beiräte, die auch die Akteure in einem Politikfeld mit einbeziehen, sind in der Regel größer und bearbeiten daher häufig eher konkrete, eng umrissene Fragestellungen. Im Fall des Expertenbeirats ging es darum eine Systemumstellung der Gesetzlichen Pflegeversicherung fachlich vorzubereiten und sicherzustellen, dass

6 Bereits in den ersten Jahren des Bundessozialhilfegesetzes (BSHG) wurde die Frage diskutiert, inwieweit ein allgemeiner Beaufsichtigungsbedarf, der in Folge von Krankheit oder Behinderung besteht, Teil der Definition von „nicht ohne Wartung und Pflege bleiben können“ (vgl. §§ 68, 69 des damaligen $\mathrm{BSHG}$ ) ist.

7 Pflege-Neuausrichtungs-Gesetz vom 23. Oktober 2012, BGBl. 2012 Teil I, S. 2246 bis 2264 .

8 Vgl. Bericht des Expertenbeirats, S. 14

9 Vgl. zusammenfassend Bericht des Expertenbeirats, S. $12 \mathrm{f}$.

$10 \mathrm{Vgl}$. Bericht des Expertenbeirats, S. $14 \mathrm{ff}$. 
der Prozess für alle Akteure erfolgreich und ressourcenschonend gelingen kann. Dabei hat der Expertenbeirat sich als Fachgremium verstanden, bei dem politische Interessen einzelner Akteure weitestgehend zurück stehen sollten.

Die Vorbereitung einer Systemumstellung durch einen Expertenbeirat war nicht trotz, sondern wegen der oben beschriebenen Komplexität des Vorhabens sinnvoll und effizient: Denn mit der Einbeziehung der Akteure in der Pflege erhöhen sich die Chancen, dass die Umstellung auf ein neues System von allen Akteuren fachlich mit befruchtet, dass das Ergebnis mit getragen wird und im Sinne der Betroffenen in der Praxis möglichst reibungslos gelingt. Zudem erfordert die Umstellung auf ein neues System auch intensive Informationsvermittlung: Alle Akteure müssen den neuen Ansatz verinnerlichen, um ihn umsetzen zu können. Den im Expertenbeirat vertretenen Fachorganisationen und -institutionen kam daher auch eine Mittler- und Multiplikatorenrolle zu, die bereits den Einstieg in die Systemumstellung markiert.

Gleichwohl gibt es Fragestellungen, bei denen fachliche Fragen nicht von sozialpolitischen Entscheidungen zu trennen sind. Ein Beispiel hierfür ist die Frage, wie die Schnittstellen zwischen der Gesetzlichen Pflegeversicherung und anderen Sozialleistungssystemen zu gestalten sind. Derartige Fragen können sinnvoll nur im Gesamtgefüge des Sozialleistungssystems gelöst werden, so dass der Expertenbeirat hier zum Teil nur Hinweise aus Sicht der Pflegeversicherung geben konnte, nicht aber alle sich stellenden Fragen klären.

Die Selbstbeschränkung des Expertenbeirats in bestimmten Fragen ist kein Manko, sondern logische Folge der zwei zentralen Vereinbarungen über seine Arbeitsweise: Einerseits, seine Entscheidungen aus seiner Fachlichkeit, nicht aus politischen Interessen heraus, zu fundieren, und andererseits, seine Empfehlungen möglichst im Konsens zu verabschieden. Daher hat der Expertenbeirat die zu beantwortenden Fragen in der Regel so weit voran getrieben, wie fachliche Begründungen reichen (können), im übrigen die möglichen Optionen mit ihren jeweiligen Auswirkungen dargestellt. Damit erhält der
Gesetzgeber nicht eine Blaupause für die Gesetzgebung zur Einführung des neuen Pflegebedürftigkeitsbegriffs, aber in Teilbereichen recht weit gediehene Empfehlungen und in anderen Bereichen eine detaillierte Aufarbeitung der möglichen Optionen als Entscheidungsgrundlage.

Abschließend sei zu diesem Punkt noch angemerkt, dass die Selbstbeschränkung des Expertenbeirats auf die Fragen, die für eine gesetzgeberische Umsetzung notwendig sind, auch eine arbeitsorganisatorische Funktion hatte: Viele in der Pflegelandschaft seit Jahren diskutierte Fragen (z.B. die Angleichung der Sachleistungshöhen für ambulante und stationäre Sachleistungen, die Frage der Verortung der medizinischen Behandlungspflege in der Kranken- bzw. Pflegeversicherung oder die Frage der Personalausstattung von Einrichtungen) werden durch die Einführung des neuen Pflegebedürftigkeitsbegriffs nicht un-

Der Expertenbeirat hat bewusst darauf verzichtet, nur mittelbar berührte Grundsatzfragen in sein bereits umfassendes Themenspektrum aufzunehmen.

mittelbar berührt oder bedürfen grundsätzlicherer Erwägungen als die, die im Rahmen der Arbeit des Expertenbeirats zeitlich möglich waren. Daher hat der Expertenbeirat bewusst und sinnvoller Weise darauf verzichtet, andere, nur mittelbar berührte Grundsatzfragen in sein bereits umfassendes Themenspektrum aufzunehmen, zumal die konsensuale Entscheidungsfindung bei vielen dieser anderen Grundsatzfragen voraussichtlich nicht möglich gewesen wäre und eher das Risiko bestanden hätte, dass auch der Konsens zum neuen Pflegebedürftigkeitsbegriff darunter gelitten hätte.

\section{Zentrale Empfehlungen und Hinweise zu den notwendigen inhaltlichen Anpassungen der Pflegeversicherung bei Einführung des neuen Pflegebedürftigkeitsbegriffs}

Nachstehend werden zentrale Empfehlungen und Hinweise des Expertenbeirats kurz vorgestellt. Dabei wird das Ge- wicht, dass die jeweiligen Themen im Expertenbeirat hatten, mit berücksichtigt.

\subsection{Das neue}

Begutachtungsassessment (NBA) im zukünftigen

Begutachtungsverfahren: Empfehlungen zur Kinderbegutachtung und besonderen Bedarfskonstellationen

Der Expertenbeirat hat insbesondere zur Kinderbegutachtung und der Frage besonderer Bedarfskonstellationen, die im Prozess der Entwicklung des NBA offen geblieben waren, ergänzende Empfehlungen erarbeitet. Das NBA ist damit grundsätzlich einführungsreif.

Mit dem NBA werden die Bedarfslagen pflegebedürftiger Kinder, die für den Lebens- und Versorgungsalltag von pflegebedürftigen Kindern und ihren Eltern von besonderer Bedeutung sind und bei der heutigen Begutachtung für die Pflegestufen größtenteils unberücksichtigt bleiben, zukünftig besser erfasst. Damit verbessert sich grundsätzlich die Einstufung pflegebedürftiger Kinder. Eine Besonderheit gibt es bei der Einstufung von Kindern von 0-18 Monaten. Diese können aufgrund der Tatsache, dass auch gesunde Kinder in diesem Alter ein großes Maß an „Unselbständigkeit“ aufweisen, nur schwer höhere Pflegegrade erreichen. Um dem Rechnung zu tragen und zugleich die ansonsten erforderlichen, häufigen Begutachtungen zu vermeiden, wird für Kinder von 0-18 Monaten eine pauschale Einstufung, die der Höhe nach zwischen Pflegegrad 2 und 3 liegen wird, empfohlen. ${ }^{11} \mathrm{Ab} 18$ Monaten werden diese Kinder dann regulär mit dem NBA begutachtet, weil bei anhaltender Unselbständigkeit dann auch weitere Module in die Bewertung einfließen und höhere Punktzahlen erreicht werden können. Es können dann regulär auch die Pflegegrade 3 und 4 erreicht werden.

Besonderheiten bestehen auch bei zwei weiteren Gruppen: Einerseits bei Menschen mit Gebrauchsunfähigkeit beider Arme und beider Beine und damit einhergehendem vollständigen Verlust der Greif-, Steh- und Gehfunktionen, andererseits bei Menschen mit ausgeprägten motorischen Verhaltenauffälligkeiten mit Selbst- und Fremdgefähr-

11 Bericht des Expertenbeirats, S. 27. 
dung, die einen sehr hohen personellen Aufwand verursachen. Der Umgang mit diesen beiden Bedarfskonstellationen steht im Kontext mit der Frage, welche Zugangskriterien für eine Einstufung in den höchsten Pflegegrad 5 gegeben sein müssen. Der Expertenbeirat hat hierzu die Empfehlung ausgesprochen, dass der Pflegegrad 5 (Personen mit einem außergewöhnlich hohem Hilfe- und Betreuungsbedarf) entweder erreicht wird

a. wenn der Schwellenwert von 90 Punkten erreicht wird oder

b. wenn unabhängig vom Schwellenwert eine Gebrauchsunfähigkeit beider Arme und beider Beine vorliegt oder

c. wenn unabhängig vom Schwellenwert ausgeprägte motorische Verhaltensauffälligkeiten mit Selbst- oder Fremdgefährdung bestehen. ${ }^{12}$

Der Expertenbeirat weist darauf hin, dass das NBA als „lernendes System“ $\mathrm{zu}$ begreifen ist und die Einführung des neuen Pflegebedürftigkeitsbegriffs durch eine wissenschaftliche Evaluation begleitet werden sollte. ${ }^{13}$

\subsection{Die Grundlage für die Bemessung der Leistungshöhen: Der Zeitaufwand für Pflege- und Betreuung allein ist kein geeigneter Maßstab}

Kern der Beratungen des Expertenbeirats war nicht - wie durch die Medienberichterstattung zum Ende der Beiratsarbeit angenommen werden könnte - die Frage, wie viele Milliarden eine Einführung des neuen Pflegebedürftigkeitsbegriffs kosten muss oder darf. Kern der Beratungen war, nach welchen Grundsätzen auf der Basis des neuen Verständnisses von Pflegebedürftigkeit die Leistungshöhen festgelegt werden sollen und können. Insbesondere die zuständige Arbeitsgruppe 1 „Leistungsrecht und Schnittstellen zu anderen Sozialleistungssystemen" hat unter Beteiligung verschiedener Wissenschaftler/innen und Disziplinen - zu dieser Frage einen intensiven fachlichen Diskurs geführt. Aus dem Selbstverständnis des Expertenbeirats heraus, dass seine Empfehlungen fachlich zu begründen sind, ggf. sogar fachwissenschaftlich bzw. auf der Basis von (repräsentativen) Studien, wurde eingehend geprüft, ob und wenn ja wie sich Leistungsbeträge fachlich begründen und/oder aus dem NBA ableiten lassen.

Im gegenwärtigen System erfolgt die Einstufung in eine Pflegestufe abhängig von der Ermittlung des Zeitaufwands für die Angehörigenpflege. Abgesehen von der Ungenauigkeit und der problematischen Anreizstruktur, die dieses Verfahren birgt, perpetuiert es die bestehenden Ungerechtigkeiten beim Leistungszu-

Diese Abkehr vom Faktor „Zeit"
als Maßstab für die Einstufung
in die neuen Pflegegrade bleibt
auch für die Leistungsbemessung
handlungsleitend.

gang: Denn es misst nur die Zeit, die für die Unterstützung bei oder Kompensation von bestimmten, katalogartig aufgeführten und inhaltlich begrenzten, körperbezogenen Verrichtungen aufgewendet wird, die insbesondere aufgrund von vorrangig körperlichen Beeinträchtigungen, d.h. Krankheiten oder Behinderungen, bestehen. Der Zeitaufwand für Betreuung sowie psychosoziale Unterstützung, der bei kognitiven oder psychischen Beeinträchtigungen im Vordergrund steht und häufig ein Vielfaches des Zeitaufwands für körperliche Pflege darstellt, bleibt hingegen unberücksichtigt.

Da die Zeitaufwände bei verschiedenen Beeinträchtigungen weder dem Ausmaß noch dem Inhalt nach (ist z.B. der Zeitaufwand für körperbezogene Pflege gleich zu setzen mit dem Zeitaufwand für ständige Präsenz? Ist ein Zeitaufwand von 1 x $10 \mathrm{~min}$. für das Waschen eines Pflegebedürftigen das gleiche wie $10 \mathrm{x} 1$ min. nachts im Schlaf unterbrochen zu werden? Ist der indirekte Zeitaufwand für die Organisation der Pflege im Hintergrund mit einzubeziehen?) vergleichbar sind, werden mit dem neuen Begutachtungsassessment nicht Zeitaufwände, sondern Schweregrade der Beeinträchtigung bzw. das Ausmaß der Selbständigkeit sowie ggf. Häufigkeiten des Erfordernisses personeller Unterstützung erfasst.

Diese Abkehr vom Faktor "Zeit" als Maßstab für die Einstufung in die neuen Pflegegrade bleibt aus logischen Gründen auch für die Leistungsbemessung handlungsleitend, da ansonsten die Ziele des neuen Pflegebedürftigkeitsbegriffs konterkariert werden könnten. Daher sieht der Expertenbeirat es nicht als sinnvoll an, Leistungsbeträge allein über z.B. eine Messung des Zeitaufwands für pflegerische und/oder betreuende Tätigkeiten zu begründen. Insbesondere in der ambulant-häuslichen Versorgung sind die Zeitaufwände aus Gründen, die nichts mit der Schwere der Pflegebedürftigkeit, sondern dem individuellen Versorgungssetting und den Präferenzen zu tun haben, so unterschiedlich, dass sich hieraus kein sinnvoller Maßstab ableiten lässt. Mangels wissenschaftlich gesicherter, objektiver Kriterien zur Bewertung des Zeitaufwands lassen sich in der ambulant-häuslichen Versorgung auch keine Relationen für „richtige“ Leistungsbeträge rechnerisch ermitteln. ${ }^{14}$ Aus Studien zum professionellen Ressourceneinsatz sind in der ambulanthäuslichen Versorgung zudem wegen des hohen Anteils der Angehörigenpflege am Gesamtpflege- und Betreuungsaufwand und des methodischen Problems, dass nur Ist-Analysen ohne Bewertung des fachlich „richtigen“ Aufwands möglich sind, nur analytische Erkenntnisse möglich. In der vollstationären Versorgung, in der die Rahmenbedingungen eher standardisiert sind, können entsprechende Studien in einem ersten Schritt zur Bildung von Äquivalenzziffern zur Bestimmung der Relationen zwischen den Leistungsbeträgen beitragen. ${ }^{15}$

Als Maßstab für die Leistungsbemessung sieht der Expertenbeirat neben dem Anstieg der Schwere der Beeinträchtigung, wie er sich aufgrund des NBA als Ausgangspunkt darstellt, daher vor allem die Auswahl und Gewichtung verschiedener fachpolitischer Zielsetzungen, die mit einer bestimmten Gestaltung des Verhältnisses der Leistungsbeträge verwirklicht werden sollen. ${ }^{16}$ Welche fachpolitischen Zielsetzungen nach seiner Auffassung relevant sein können, hat er im Bericht dargelegt. An die verschiedenen Ansätze zur Leistungsbemessung hat er Rechenbeispiele angeknüpft, die exemplarisch zeigen, welche Auswirkungen verschiedene Ansätze der Spreizung von Leistungsbeträgen unter Berücksichtigung verschie-

12 Bericht des Expertenbeirats, S. 25

13 Bericht des Expertenbeirats, S. 26.

14 Bericht des Expertenbeirats, S. 45.

15 Bericht des Expertenbeirats, S. 46, 47.

16 Bericht des Expertenbeirats, S. 47. 
dener, vom Gesetzgeber festzulegender Leistungsniveaus haben können.

Eine Ausnahme stellt insoweit die leistungsrechtliche Hinterlegung des zukünftigen Pflegegrads 1 dar. Hierfür wird vom Expertenbeirat in der ambulant-häuslichen Versorgung ein Leistungsbetrag von 100 EUR empfohlen, der für Sach- und Entlastungsleistungen im Wege der Kostenerstattung flexibel verwendet werden kann. ${ }^{17}$

\subsection{Die Suche nach der "magischen Zahl“: Welche „notwendigen“" Mehrausgaben ergeben sich für die Soziale Pflegeversicherung?}

Auch aus der Zusammenschau der im Bericht dargestellten Rechenbeispiele ergibt sich keine konkrete Summe, die aus fachlicher Sicht eindeutig für die Umsetzung des neuen Pflegebedürftigkeitsbegriffs „erforderlich“ ist. Festgehalten werden kann lediglich, dass bei einer „technischen“ Umsetzung, d.h. einer schematischen Übertragung der bisherigen Leistungsbeträge auf die neuen Pflegegrade unter Einbeziehung der bereits durch das PNG in die Pflegeversicherung geflossenen Mittel, weitere Mehrausgaben von ca. 2 - 2,5 Mrd. EUR anfallen würden. ${ }^{18} \mathrm{Da}$ diese ,technische“ Umsetzung jedoch weder den Anstieg der Schwere der Betroffenheit, wie er sich im NBA darstellt, noch explizit fachpolitischen Zielsetzungen berücksichtigt, kann sie nur als sehr grober Anhaltspunkt für zukünftige Mehrausgaben dienen; diese können je nach konkreter fachpolitischer Ausgestaltung deutlich variieren. Sieht man es - wie es vom Expertenbeirat weit überwiegend favorisiert wird - als gesetzt an, dass sich die zukünftigen Leistungsbeträge das bisherige Leistungsniveau nicht unterschreiten sollen, wäre eine Umsetzung, deren Ausgabenbetrag unter dieser Summe liegt, wohl kaum möglich.

Hinzu kommt, dass die vom Expertenbeirat betrachtete Fragestellung der Umsetzung des neuen Pflegebedürftigkeitsbegriffs andere pflegepolitische Diskussionen, die ebenfalls ausgabenrelevant sein können, wie oben erläutert bewusst außen vor lässt. Wie mit diesen umgegangen werden soll, ist ebenfalls im politischen Prozess zu klären, bevor zuverlässige Ausgabenabschätzungen erfolgen können. Dies erklärt auch die z.T. sehr unterschiedlichen Aussagen darüber, welche Mehrausgaben in der Sozialen Pflegeversicherung bei Einführung des neuen Pflegebedürftigkeitsbegriffs „notwendig“ sind. Fachpolitisch ist es nach Auffassung der Autorin in jedem Fall sinnvoll, die Einführung des neuen

\section{Fachpolitisch ist es sinnvoll, die Einführung des neuen Pflegebedürftigkeitsbegriffs nicht auf die Umsetzung der Beiratsergebnisse zu beschränken.}

Pflegebedürftigkeitsbegriffs nicht auf die Umsetzung der Beiratsergebnisse zu beschränken, sondern mit anderen Entwicklungen in der Pflege zu verzahnen.

\subsection{Sonderleistungen ade: $\mathrm{Zu}$ einem neuen System der Pflege- und Entlastungsleistungen für alle}

Neben der fachlichen Grundlage für die Leistungsbemessung hatte der Expertenbeirat die Frage zu klären, wie der Übergang von einem Leistungsrecht, dass aus Regelleistungen für insbesondere körperlich beeinträchtigte Pflegebedürftige und sukzessive eingeführten und immer weiter ausdifferenzierten Sonderleistungen für Personen mit eingeschränkter Alltagskompetenz (PEA) nach $\mathbb{S} 45$ a SGB XI besteht, zu einem einheitlich gestalteten Leistungsrecht für alle zukünftig Pflegebedürftigen erfolgen soll. Prämisse ist, dass die für kognitiv und psychisch beeinträchtigte Pflegebedürftige so wichtige Betreuung zukünftig regelhafte „3. Säule“ der Pflegeversicherung wird und keine nachrangige oder untergeordnete Leistungsart. ${ }^{19}$

Dabei spricht sich der Expertenbeirat in seinem Abschlussbericht grundsätzlich dafür aus, die bestehenden Sonderleistungen für PEA hinsichtlich ihrer Leistungsvolumina ins Regelleistungssystem zu integrieren. ${ }^{20}$ Dies soll allerdings nicht schematisch, sondern unter konzeptioneller Weiterentwicklung des Leistungsrechts unter Berücksichtigung der Zwecke, die mit der jeweiligen Leistung erreicht werden sollen, erfolgen. $\mathrm{Zu}$ diesem Zweck sollen die Leistungen in der ambulant-häuslichen Versorgung zu- künftig in zwei große Gruppen eingeteilt werden ${ }^{21}$ : Leistungen zur Kompensation von Beeinträchtigungen und Leistungen zur Entlastung der Pflegebedürftigen und ihrer Angehörigen. Zur Kompensation von Beeinträchtigungen dienen vor allem die Leistungen der Pflegesachleistung, $\mathbb{S} 36$ SGB XI. Die in $\$ 124$ SGB XI befristet vorgenommene Ausweitung der Sachleistung auf pflegerische Betreuungsmaßnahmen soll dabei nach Empfehlung des Expertenbeirats zur Regelleistung des $\$ 36$ SGB XI werden. Das bedeutet, dass kognitiv und psychisch beeinträchtigte Pflegebedürftige zukünftig einen Sachleistungsanspruch in gleicher Höhe haben wie körperlich beeinträchtigte Pflegebedürftige.

Zur Entlastung der Pflegebedürftigen und ihrer Angehörigen soll daneben eine neue Entlastungsleistung geschaffen werden, die zunächst ähnlich strukturiert ist wie die bisherigen zusätzlichen Betreuungsleistungen nach \45b SGB XI, nunmehr jedoch allen Pflegebedürftigen zur Verfügung steht. Die Entlastungsleistung ist - je nach fachpolitischer Zielsetzung - modular erweiterbar um weitere Leistungen der Pflegeversicherung und bietet daher über den Status Quo hinaus Chancen für eine flexiblere, individuelle Gestaltung und eine verbesserte Entlastung von Pflegeund Betreuungsarrangements.

In der vollstationären Versorgung war es dem Expertenbeirat ein besonderes Anliegen, den erreichten Status Quo an (zusätzlicher) Betreuung und Aktivierung, der durch die Einführung von $\mathbb{S} 87 \mathrm{~b}$ SGB XI erreicht wurde, zu erhalten. ${ }^{22}$ Aus der Prämisse, dass alle zukünftig Pflegebedürftigen Zugang zu den gleichen Leistungen haben müssen, folgt nach Ansicht des Expertenbeirats, dass diese Leistungen zukünftig allen

\section{Bericht des Expertenbeirats, S. 42.}

18 Dies deckt sich, wenn man die ca. 1 Mrd. EUR, die bereits mit dem PNG in die Soziale Pflegeversicherung geflossen sind, hinzu rechnet, grob mit der Summe für das bis auf den Schwellenwert des Pflegegrads 1 vergleichbare Szenario „1A“ des Beirats 2009, bei der bei einer solchen „technischen" Umsetzung ca. 3,4 Mrd. Mehrausgaben ermittelt hatte.

19 Bericht des Expertenbeirats, S. 32.

20 Bericht des Expertenbeirats, S. 34.

21 Bericht des Expertenbeirats, S. 34.

22 Bericht des Expertenbeirats, S. 37. 
Pflegebedürftigen zur Verfügung gestellt werden müssen. Die von ihm vorgeschlagenen Umsetzungsvarianten stellen alle sicher, dass hier kein Rückschritt hinter den erreichten Status Quo erfolgt.

\subsection{Ein schwieriges Verhältnis: Neue Leistungsinhalte und Schnittstellen zu anderen Sozialleistungssystemen}

Neben der Neugestaltung der Leistungsbeträge und der Relationen zwischen den fünf Pflegegraden hat sich der Expertenbeirat mit der Frage befasst, inwiefern sich mit dem neuen Verständnis von Pflegebedürftigkeit auch die Leistungsinhalte verändern (müssen). Konsens besteht darüber, dass die Leistungsinhalte grundsätzlich mit dem neuen Begriff der Pflegebedürftigkeit korrespondieren müssen ${ }^{23}$. Daher wird Betreuung zukünftig als regelhafter Bestandteil („3. Säu$\mathrm{le}^{\text {“) }}$ der Pflegeleistungen angesehen. Im Detail gestaltet sich die Festlegung der Leistungsinhalte allerdings noch deutlich komplexer, insbesondere dann, wenn man möglichst klare Grenzziehungen zu den Leistungen anderer Sozialleistungssysteme schaffen will.

Auf Bitte des Expertenbeirats haben Dr. Wingenfeld \& Dr. Gansweid ${ }^{24}$ in ergänzender Auswertung ihrer Studie auch aufgezeigt, welches Spektrum an „psychosozialer Unterstützung “ in der ambulant-häuslichen Versorgung existiert. Diese Darstellung sollte Ausgangspunkt

\section{Es ist der Wunsch der Mehrheit der Mitglieder im Expertenbeirat, dass der Startschuss für die Einführung eines neuen Pflegebedürftigkeitsbegriffs nach der BT-Wahl die Priorität bekommt, die sie verdient.}

für die vom Expertenbeirat empfohlene weitere Vertiefung der Frage, welche Leistungsinhalte zukünftig als „Betreuung“ von der Pflegeversicherung finanziert werden sollen, sein. Eine Lösung dieser Frage ist realistischer Weise aber wohl nur bei gleichzeitiger Betrachtung der (z.T. fachlich, z.T. politisch) festgelegten Finanzierungszuständigkeiten für gleichgelagerte oder ähnliche Leistungen anderer Sozialleistungssysteme möglich. Daher konnte der Expertenbeirat diese Frage nicht abschließend beraten. Damit war auch keine eindeutige Empfehlung dazu möglich, wie die Schnittstellen insbesondere zur Eingliederungshilfe für behinderte Menschen zukünftig zu gestalten seien, so dass der Expertenbeirat sich zu diesem Aspekt auf grundsätzliche Hinweise beschränkt hat.

Zur Frage der Schnittstelle zur Hilfe zur Pflege geht der Expertenbeirat davon aus, dass der zukünftige Begriff der Pflegebedürftigkeit im SGB XI und SGB XII identisch sein wird, so dass Leistungen der Hilfe zur Pflege insbesondere dann erforderlich werden können, wenn Pflegebedürftigkeit für voraussichtlich weniger als 6 Monate besteht oder ein Bedarf an Pflegeleistungen besteht, der über die Leistungen der Pflegeversicherung hinaus geht. ${ }^{25}$ Auch unterhalb des Schwellenwerts von 15 Punkten (Eingangsschwellenwert für das Erreichen des zukünftigen Pflegegrads 1) besteht im pflegewissenschaftlichen Sinne ein geringfügiger personeller Hilfebedarf. ${ }^{26}$

\subsection{Gestaltung des Systemübergangs: Formale Überleitung und Bestandsschutz}

Zur Frage, wie der Übergang von einem Begutachtungssystem mit drei Stufen, das auch in vielen Rechtsnormen des SGB XI in Bezug genommen wird, zu einem inhaltlich anders aufgebauten Begutachtungssystem mit fünf Pflegegraden gelingen kann, hat der Expertenbeirat auf verschiedenen Ebenen Stellung genommen. Dort, wo Rechtsnormen bisher an die drei Stufen anknüpfen, empfiehlt er in der Regel die Übertragung auf die fünf Pflegegrade nach einem formalen Schema. ${ }^{27}$ Für die kollektive Überleitung der bisherigen Leistungsbezieher empfiehlt er ebenfalls dieses für die Betroffenen unbürokratische Schema, mit dem sich nach der vom Expertenbeirat favorisierten Variante die Mehrzahl der Leistungsbezieher in der ambulant-häuslichen Versorgung zukünftig zudem besser stellen würde als bisher. ${ }^{28}$

Für diejenigen, die nach der kollektiven Überleitung weniger Leistungen erhalten würden als bisher, wird ein
Bestandsschutz in Höhe der bisherigen Leistungen empfohlen, der sich nach Auffassung des Expertenbeirats auch auf nach dem Überleitungstermin getroffene Änderungen im Versorgungsarrangement beziehen soll. ${ }^{29}$ Neue Leistungsbezieher werden nach dem Stichtag automatisch nur nach dem NBA eingestuft. Eine Parallelität von bisherigem und neuem Begutachtungsverfahren kann damit vermieden werden, ebenso wie umfangreiche Neubegutachtungen.

\section{Der Fahrplan für die Einführung des neuen Pflegebedürftigkeitsbegriffs}

Der Expertenbeirat sieht im Anschluss an das Gesetzgebungsverfahren ab Inkrafttreten eines Gesetzes, dass die Leistungsgewährung aufgrund eines neuen Pflegebedürftigkeitsbegriffs regelt, einen Zeitraum von bis zu 18 Monaten als (ambitionierte) Vorlaufzeit als erforderlich an, um die notwendigen (auch untergesetzlichen) Vorbereitungsarbeiten, z.B. eine neue Begutachtungsrichtlinie oder die Anpassung von Vereinbarungen und Verträgen, vor bzw. bis zum Stichtag zu erledigen. ${ }^{30}$

\section{Zusammenfassung und Fazit}

Der Expertenbeirat hat mit seinem Abschlussbericht wesentliche Grundfragen geklärt, die sich vor Einführung eines neuen Pflegebedürftigkeitsbegriffs stellen. Neben der Einführung des neuen Pflegebedürftigkeitsbegriffs sind in der Pflege jedoch noch zahlreiche weitere Fragen zu klären. Es ist der Wunsch der Mehrheit der Mitglieder des Expertenbeirats, dass die lang erwartete Einführung als Startschuss für ein modernes, pflegefachlich fundiertes Pflegesystem bei der Bundestagswahl und den anschließenden Koalitionsverhandlungen die Priorität eingeräumt bekommt, die sie verdient.

23 Bericht des Expertenbeirats, S. 32.

24 Bericht des Expertenbeirats, S. 32.

25 Bericht des Expertenbeirats, S. 70.

26 Bericht des Expertenbeirats, S. 70.

27 Bericht des Expertenbeirats, S. 83, unter Bezugnahme auf Tabelle 32 auf S. 84 .

28 Für die Optionen, die in der vollstationären Versorgung bestehen, vgl. Bericht des Expertenbeirats, S. 90f.

29 Bericht des Expertenbeirats, S. $87 \mathrm{ff}$

30 Bericht des Expertenbeirats, S. 92 f. 